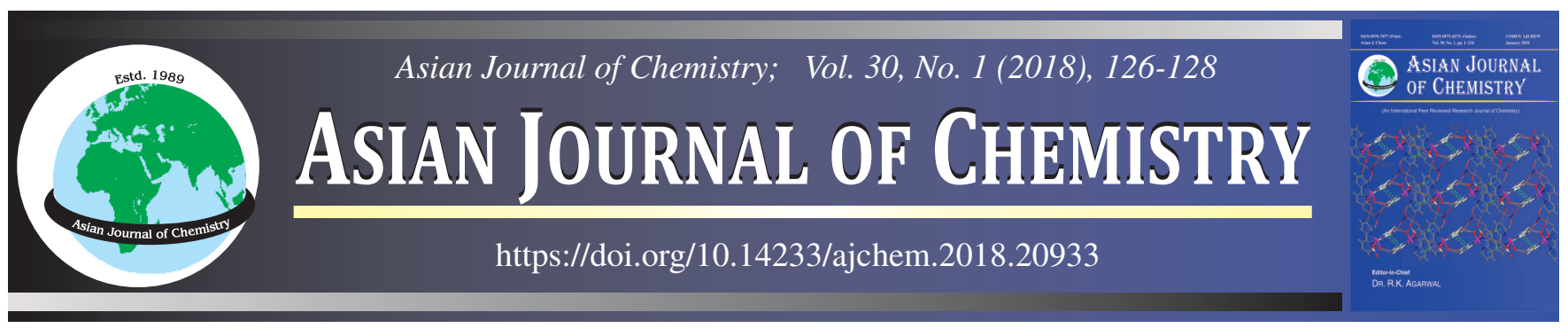

\title{
Synthesis and Antibacterial Study of Thymol Derivatives
}

Nurul Hazwani Che Abdul Rahim ${ }^{1}$, AsnuZilawati Asari ${ }^{1, *}$, Noraznawati Ismail ${ }^{2}$, Fauziah Abdullah ${ }^{3}$, Hasnah Osman $^{4}$ and Soraya Shafawati Mohamad Tahier ${ }^{1}$

\begin{abstract}
${ }^{1}$ School of Fundamental Science, , Universiti Malaysia Terengganu, 21030 Kuala Terengganu, Terengganu, Malaysia ${ }^{2}$ Institute of Marine Biotechnology, Universiti Malaysia Terengganu, 21030 Kuala Terengganu, Terengganu, Malaysia ${ }^{3}$ Phytochemistry Programme, Natural Product Division, Forest Research Institute Malaysia, 52109 Kepong, Selangor, Malaysia ${ }^{4}$ School of Chemical Science, Universiti Sains Malaysia, 11800 Pulau Pinang, Malaysia

*Corresponding author: E-mail: asnu@umt.edu.my
\end{abstract}

Five thymol derivatives (2-6) were synthesized and evaluated for their antibacterial activity against four bacterial strains i.e., Bacillus subtilis, Staphylococcus aureus, Staphylococcus epidermidis and Escherichia coli using standard well-diffusion method.

Keywords: Thymol derivatives, Antibacterial activity, Well-diffusion method.

\section{INTRODUCTION}

Thymol (2-isopropyl-5-methylphenol) (1) is naturally occurring phenolic monoterpene derivative of cymene, which is found in essential oils extracted from plants belonging to the Lamiaceae family [1]. Since 16th century, thymol-rich essential oils have been evaluated for their benefits in medicinal application [2,3] as well as for their antimicrobial properties $[1,4]$. Thymol (1) itself exhibits a large number of biological activities, such as antibacterial [5], antileishmanial [6], antiinflammatory [7], antitumor [8] and aedes aegypti larvicidal [9] properties. Thymol also exhibited insecticidal and genotoxic activities on Drosophila melanogaster [10].

In continuation of our interest in searching for potential antibacterial compounds derived from natural products [11], herein we report the synthesis, characterization and antibacterial evaluation of thymol esters and ethers (2-6) using well-diffusion method.

\section{EXPERIMENTAL}

Thymol is commercially available and purchased from Sigma-Aldrich. All of other reagents were obtained from SigmaAldrich, Merck or Acros Organics and used without additional purification. All the reactions were performed under nitrogen atmosphere. The reactions were monitored by thin layer chromatography (TLC) using plastic precoated sheets (Silica gel $60 \mathrm{~F}_{254}$, $0.25 \mathrm{~mm}$ thick). Plates were visualized under UV $365 \mathrm{~nm}$ and UV $254 \mathrm{~nm}$ without treatment. Column chromatography was performed on silica gel 60 (230-400 mesh, Merck). NMR data were recorded in $\mathrm{CDCl}_{3}$ on Bruker FT-400 (400 MHz) or Jeol (500 MHz) Spectrometer. ${ }^{1} \mathrm{H}$ and ${ }^{13} \mathrm{C}$ NMR chemical shifts $(\delta)$ are given in ppm. Infrared spectra were recorded in $\mathrm{KBr}$ disc on Perkin Elmer 100 FT-IR Spectrometer. UV-visible spectra were recorded on Shimadzu UV-1601PC Spectrophotometer. HREIMS were recorded on LTQ Orbitrap mass spectrometer (Thermo Scientific).

General method for the synthesis of thymol ethers (2,3): Thymol ethers were synthesized according to the procedure described previously [11]. $\mathrm{K}_{2} \mathrm{CO}_{3}(9.99 \mathrm{mmol})$ was added to a solution of thymol $(6.66 \mathrm{mmol})$ and benzyl halide $(9.99 \mathrm{mmol})$ in acetone $(7 \mathrm{~mL})$ under an inert atmosphere. The mixture was refluxed upon completion via TLC monitoring. After the completion, the reaction crude was diluted with $30 \mathrm{~mL}$ of distilled water and then extracted with $\mathrm{CH}_{2} \mathrm{Cl}_{2}(3 \times 30 \mathrm{~mL})$. The organic extracts were dried over anhydrous $\mathrm{MgSO}_{4}$ and concentrated under reduced pressure. The residue was purified by column chromatography (hexane:chloroform) to give compounds $(\mathbf{2 , 3})$.

General method for the synthesis of thymol esters (4-6): Triethylamine $(5 \mathrm{~mL})$ was added to a solution of thymol (6.60 $\mathrm{mmol})$ in dichloromethane $(15 \mathrm{~mL})$. The mixture was stirred for $0.5 \mathrm{~h}$ at $0{ }^{\circ} \mathrm{C}$. Then, an excess of acyl chloride (26.62 mmol) was added dropwise. The solution was stirred for another 30 min at $0{ }^{\circ} \mathrm{C}$ before it slowly warmed to room temperature and continued stirring for another $24 \mathrm{~h}$. The progress of the reaction was monitored by thin layer chromatography (TLC). After the completion of reaction, the solvent was removed via in vacuo. The residue was purified by column chromatography (hexane: chloroform) to yield compounds 4-6 in good yields. 
2-(Benzyloxy)-1-isopropyl-4-methylbenzene (2) [12]: Yield: $84.71 \%$; IR $\left(\mathrm{KBr}, \mathrm{v}_{\max }, \mathrm{cm}^{-1}\right)$ : 3032, 2960, 1612, 1455, 1256; UV (MeOH) $\lambda_{\max }(\log \varepsilon) 281.0$ (3.5), 274.5 (3.5) nm; ${ }^{1} \mathrm{H}$ NMR $\left(400 \mathrm{MHz}, \mathrm{CDCl}_{3}\right) \delta 1.22\left(\mathrm{~d} J=7.2 \mathrm{~Hz}, 6 \mathrm{H}, 2 \mathrm{CH}_{3}\right.$, H-6), 2.31 (s, 3H, $\left.\mathrm{CH}_{3}, \mathrm{H}-1\right), 3.33-3.40$ (m, 1H, H-5), 5.06 (s, 2H, $\left.\mathrm{CH}_{2}, \mathrm{H}-7\right), 6.77$ (d, J = 8.0 Hz, 1H, $\left.\mathrm{CH}_{\mathrm{ar}}\right), 6.74(\mathrm{~s}, 1 \mathrm{H}$, $\mathrm{CH}_{\mathrm{ar}}$ ), $7.13\left(\mathrm{~d}, J=7.6 \mathrm{~Hz}, 1 \mathrm{H}, \mathrm{CH}_{\mathrm{ar}}\right), 7.31-7.46\left(\mathrm{~m}, 5 \mathrm{H}, \mathrm{CH}_{\mathrm{ar}}\right.$ ); ${ }^{13} \mathrm{C} \mathrm{NMR}\left(100 \mathrm{MHz}, \mathrm{CDCl}_{3}\right) \delta 21.3,22.8,26.6,9.9,112.6$, $121.4,125.9,127.1,127.6,128.5,134.0,136.3,137.6,155.8$ ppm. EIMS $[\mathrm{M}]^{+} m / z=240$.

2-Isopropyl-4-methyl-2-[(4-nitrobenzyl)oxy]benzene (3): Yield: $84.59 \%$; IR (KBr, $\left.v_{\max }, \mathrm{cm}^{-1}\right)$ : 3071, 2962, 1606, $1517,1453,1342,1262$; UV (MeOH) $\lambda_{\max }(\log \varepsilon) 269.5$ (4.0), $211.5(4.2) \mathrm{nm} ;{ }^{1} \mathrm{H}$ NMR (400 MHz, $\left.\mathrm{CDCl}_{3}\right) \delta 1.25(\mathrm{~d} J=6.8$ $\left.\mathrm{Hz}, 6 \mathrm{H}, 2 \mathrm{CH}_{3}, \mathrm{H}-6\right), 2.31$ (s, 3H, $\left.\mathrm{CH}_{3}, \mathrm{H}-1\right), 3.33-3.40$ (m, 1H, H-5), 5.17 (s, 2H, $\left.\mathrm{CH}_{2}, \mathrm{H}-7\right), 6.68\left(\mathrm{~s}, 1 \mathrm{H}, \mathrm{CH}_{\mathrm{ar}}\right) 6.81$ (d, J $\left.=76 \mathrm{~Hz}, 1 \mathrm{H}, \mathrm{CH}_{\mathrm{ar}}\right), 7.16\left(\mathrm{~d}, J=7.6 \mathrm{~Hz}, 1 \mathrm{H}, \mathrm{CH}_{\mathrm{ar}}\right), 7.63(\mathrm{~d}, J$ $\left.=8.8 \mathrm{~Hz}, 2 \mathrm{H}, \mathrm{CH}_{\mathrm{ar}}\right), 8.27\left(\mathrm{~d}, J=8.8 \mathrm{~Hz}, 2 \mathrm{H}, \mathrm{CH}_{\mathrm{ar}}\right) \mathrm{ppm} ;{ }^{13} \mathrm{C}$ NMR $\left(100 \mathrm{MHz}, \mathrm{CDCl}_{3}\right) \delta 21.3,22.8,26.6,68.7,112.5,122.0$, $123.8,126.2,127.3,134.3,126.5,145.1,147.4,155.1 \mathrm{ppm}$. EIMS $[\mathrm{M}]^{+} m / z=285$.

2-Isopropyl-5-methylphenyl 4-chlorobenzoate (4): Yield: $66.44 \%$; IR (KBr, $\left.v_{\max }, \mathrm{cm}^{-1}\right): 3029,2963,1738,1594$, 1487, 123, 753; UV (MeOH) $\lambda_{\max }(\log \varepsilon) 242.5$ (4.4) nm; ${ }^{1} \mathrm{H}$ NMR (400 MHz, $\left.\mathrm{CDCl}_{3}\right) \delta 1.21\left(\mathrm{~d} J=7.2 \mathrm{~Hz}, 6 \mathrm{H}, 2 \mathrm{CH}_{3}, \mathrm{H}-\right.$ 6), 2.34 (s, 3H, $\mathrm{CH}_{3}, \mathrm{H}-1$ ), 2.98-3.05 (m, 1H, H-5), 6.93 (s, $\left.1 \mathrm{H}, \mathrm{CH}_{\mathrm{ar}}\right), 7.08$ (d, $\left.J=7.6 \mathrm{~Hz}, 1 \mathrm{H}, \mathrm{CH}_{\mathrm{ar}}\right), 7.25(\mathrm{~d}, J=7.2 \mathrm{~Hz}$, $\left.1 \mathrm{H}, \mathrm{CH}_{\mathrm{ar}}\right), 7.50\left(\mathrm{~d}, J=8.4 \mathrm{~Hz}, 2 \mathrm{H}, \mathrm{CH}_{\mathrm{ar}}\right), 8.16(\mathrm{~d}, J=8.4 \mathrm{~Hz}$, $\left.2 \mathrm{H}, \mathrm{CH}_{\mathrm{ar}}\right) \mathrm{ppm} ;{ }^{13} \mathrm{C} \mathrm{NMR}\left(100 \mathrm{MHz}, \mathrm{CDCl}_{3}\right) \delta 20.8,23.0$, 27.3, 122.7, 126.5, 127.3, 128.0, 129.0, 131.5 136.7, 140.1, 147.9, 164.5 ppm. EIMS $[\mathrm{M}]^{+} \mathrm{m} / z=288$.

2-Isopropyl-5-methylphenyl 4-bromobenzoate (5): Yield: $94.21 \%$; IR (KBr, $\left.v_{\max }, \mathrm{cm}^{-1}\right): 3029,2962,1743,1590$, 1485, 1264; UV (MeOH) $\lambda_{\text {max }}(\log \varepsilon) 247.0$ (4.2) nm; ${ }^{1} \mathrm{H}$ NMR $\left(400 \mathrm{MHz}, \mathrm{CDCl}_{3}\right) \delta 1.21\left(\mathrm{~d} J=7.2 \mathrm{~Hz}, 6 \mathrm{H}, 2 \mathrm{CH}_{3}, \mathrm{H}-6\right), 2.34$ (s, 3H, $\left.\mathrm{CH}_{3}, \mathrm{H}-1\right), 2.97-3.04$ (m, 1H, H-5), 6.92 (s, 1H, $\mathrm{CH}_{\mathrm{ar}}$ ), $7.08\left(\mathrm{~d}, J=7.6 \mathrm{~Hz}, 1 \mathrm{H}, \mathrm{CH}_{\mathrm{ar}}\right), 7.25\left(\mathrm{~d}, J=8.0 \mathrm{~Hz}, 1 \mathrm{H}, \mathrm{CH}_{\mathrm{ar}}\right)$, $7.67\left(\mathrm{~d}, J=8.4 \mathrm{~Hz}, 2 \mathrm{H}, \mathrm{CH}_{\mathrm{ar}}\right), 8.08\left(\mathrm{~d}, J=8.7 \mathrm{~Hz}, 2 \mathrm{H}, \mathrm{CH}_{\mathrm{ar}}\right)$ ppm; ${ }^{13} \mathrm{C}$ NMR $\left(100 \mathrm{MHz}, \mathrm{CDCl}_{3}\right) \delta 20.8,23.0,27.3,122.7$, $126.5,127.3,128.5,128.8,131.6,132.0,136.7,137.0,147.9$, $164.7 \mathrm{ppm}$. EIMS $[\mathrm{M}]^{+} \mathrm{m} / z=332$.

2-Isopropyl-5-methylphenyl 4-ethylbenzoate (6):Yield: $62.35 \%$; IR (KBr, $\left.v_{\max }, \mathrm{cm}^{-1}\right): 3032,2965,1737,1611,1456$, 1237; UV (MeOH) $\lambda_{\max }(\log \varepsilon) 240.5$ (4.2) nm; ${ }^{1} \mathrm{H}$ NMR (400 $\left.\mathrm{MHz}, \mathrm{CDCl}_{3}\right) \delta 1.21\left(\mathrm{~d} J=6.8 \mathrm{~Hz}, 6 \mathrm{H}, 2 \mathrm{CH}_{3}, \mathrm{H}-6\right), 1.29$ (t, $J$ $\left.=7.6 \mathrm{~Hz}, 3 \mathrm{H}, \mathrm{CH}_{3}, \mathrm{H}-10\right), 2.34$ (s, 3H, $\left.\mathrm{CH}_{3}, \mathrm{H}-1\right), 2.72-2.77$ (m, 2H, $\left.\mathrm{CH}_{2}, \mathrm{H}-9\right), 3.00-3.10$ (m, 1H, H-5), 6.93 (s, 1H, $\mathrm{CH}_{\mathrm{ar}}$ ), $7.06\left(\mathrm{~d}, J=8.0 \mathrm{~Hz}, 1 \mathrm{H}, \mathrm{CH}_{\mathrm{ar}}\right), 7.24\left(\mathrm{~d}, J=8.4 \mathrm{~Hz}, 1 \mathrm{H}, \mathrm{CH}_{\mathrm{ar}}\right)$, $7.35\left(\mathrm{~d}, J=8.4 \mathrm{~Hz}, 2 \mathrm{H}, \mathrm{CH}_{\mathrm{ar}}\right), 8.14\left(\mathrm{~d}, J=8.0 \mathrm{~Hz}, 2 \mathrm{H}, \mathrm{CH}_{\mathrm{ar}}\right)$ ppm; $\left.{ }^{13} \mathrm{C} \mathrm{NMR} \mathrm{(100} \mathrm{MHz,} \mathrm{CDCl}_{3}\right) \delta 15.2,20.8,23.0,27.3$, 29.0, 122.9, 126.4, 127.6, 127.9, 128.1, 130.3, 136.6, 137.2, $148.2,150.5,165.4$ ppm. EIMS $[\mathrm{M}]^{+} \mathrm{m} / z=282$.

Antibacterial assay: All the synthesized compounds were evaluated for their antibacterial activity against four bacterial strains of Gram-positive and Gram-negative bacteria; three Gram-positive bacteria (Bacillus subtilis ATCC11774, Staphylococcus aureus ATCC25923 and Staphylococcus epidermidis ATCC13518) and one Gram-negative bacterium (Escherichia coli ATCC11775) using standard well-diffusion method. The Mueller-Hinton agar (MHA) plates were inoculated with cultured bacterial strains using cotton swab. By using sterile cork borer, wells of $6.0 \mathrm{~mm}$ diameter were cut on the media and loaded with $60 \mu \mathrm{L}$ of diluted compounds. $1 \mathrm{mg} / \mathrm{mL}$ of synthesized compounds were prepared in methanol. Streptomycin (Abtek Biologicals Ltd.) was used as positive control and methanol as negative control. All plates were incubated at $37^{\circ} \mathrm{C}$ for overnight before evaluating the antibacterial activity by measuring the diameter of inhibition zones against bacteria.

\section{RESULTS AND DISCUSSION}

The synthetic route for thymol derivatives $\mathbf{2 - 6}$ is illustrated in Scheme-I. By employing the previous published method [11], thymol ethers $(\mathbf{2 , 3})$ have been synthesized by the reaction of thymol (1) with benzyl halide in the presence of $\mathrm{K}_{2} \mathrm{CO}_{3}$. Whilst, the treatment of thymol (1) with acyl chloride in $\mathrm{CH}_{2} \mathrm{Cl}_{2}$ in the presence of $\mathrm{Et}_{3} \mathrm{~N}$ [11] furnished thymol esters (4-6) in $66-94 \%$ yields.

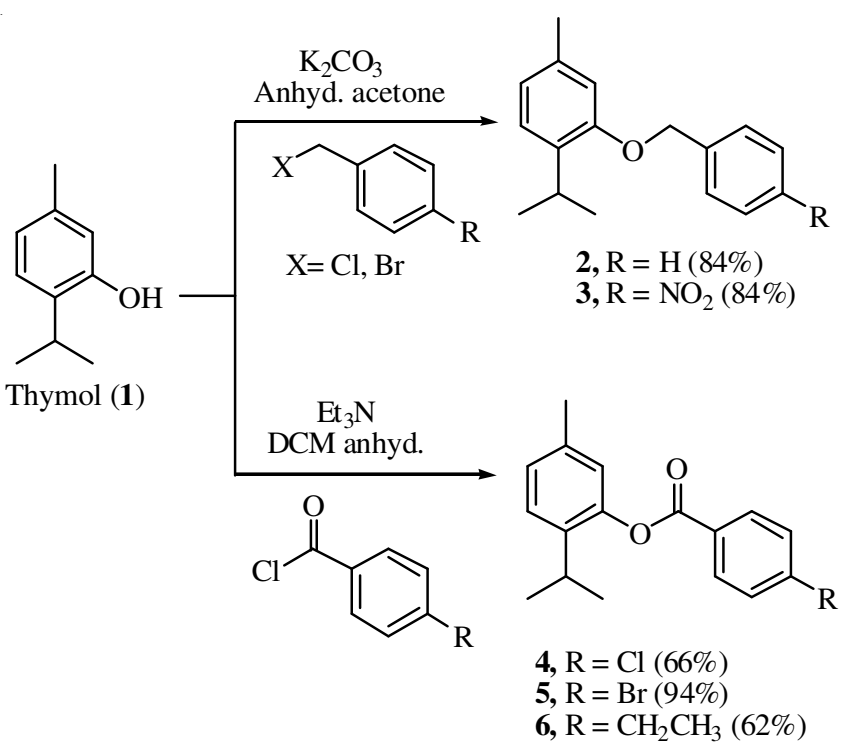

Scheme-I: Synthesis of derivatives (2-6)

All the derivatives (2-6) were screened for their antibacterial activity [11-13]. Unfortunately, when compared with standard drug as streptomycin, all the derivatives (2-6) do not show any antibacterial activity against all four types of bacteria.

\section{Conclusion}

In the present work, five thymol derivatives were synthesized and characterized by spectral studies. All the synthesized compounds were evaluated for their antibacterial activities against Bacillus subtilis, Staphylococcus aureus, Staphylococcus epidermidis and Escherichia coli using standard well-diffusion method. However, all the derivatives (2-6) did not show any antibacterial activity against all the tested strains.

\section{ACKNOWLEDGEMENTS}

The authors acknowledge Ministry of Higher Education Malaysia for financial support (RACE - 50068). 


\section{REFERENCES}

1. A. Marchese, I.E. Orhan, M. Daglia, R. Barbieri, A. Di Lorenzo, S.F. Nabavi, O. Gortzi, M. Izadi and S.M. Nabavi, Food Chem., 210, 402 (2016); https://doi.org/10.1016/j.foodchem.2016.04.111.

2. A.A.E. Ibrahim, Am. J. Biol. Life Sci., 2, 154 (2014).

3. A. Özkan and A. Erdogan, Turk. J. Biol., 35, 735 (2011) https://doi.org/10.3906/biy-1011-170.

4. M. Boskovic, N. Zdravkovic, J. Ivanovic, J. Janjic, J. Djordjevic, M Starcevic and M.Z. Baltic, Procedia Food Sci., 5, 18 (2015); https://doi.org/10.1016/j.profoo.2015.09.005.

5. N. Didry, L. Dubreuil and M. Pinkas, Pharm. Acta Helv., 69, 25 (1994); https://doi.org/10.1016/0031-6865(94)90027-2.

6. S.M. de Morais, N.S. Vila-Nova, C.M.L. Bevilaqua, F.C. Rondon, C.H. Lobo, A. de Alencar Araripe Noronha Moura, A.D. Sales, A.P.R. Rodrigues, J.R. de Figuereido, C.C. Campello, M.E. Wilson and H.F. de Andrade Jr., Bioorg. Med. Chem., 22, 6250 (2014); https://doi.org/10.1016/j.bmc.2014.08.020.
7. K.R. Riella, R.R. Marinho, J.S. Santos, R.N. Pereira-Filho, J.C. Cardoso, R.L.C. Albuquerque-Junior and S.M. Thomazzi, J. Ethnopharmacol., 143, 656 (2012); https://doi.org/10.1016/j.jep.2012.07.028.

8. A. Anderson, Int. J. Toxicol., 25, 29 (2006); https://doi.org/10.1080/10915810600716653.

9. V.B. Silva, D.L. Travassos, A. Nepel, A. Barison, E.V. Costa, L. Scotti, M.T. Scotti, R.J.B. Mendonca-Junior, R.C. Santos and S.C. Cavalcanti, J. Arthropod Borne Dis., 11, 315 (2017);

10. I. Karpouhtsis, E. Pardali, E. Feggou, S. Kokkini, Z.G. Scouras and P. Mavragani-Tsipidou, J. Agric. Food Chem., 46, 1111 (1998); https://doi.org/10.1021/jf970822o

11. N.H.C. Abdul Rahim, A. Asari, N. Ismail and H. Osman, Asian J. Chem., 29, 22 (2017); https://doi.org/10.14233/ajchem.2017.20100.

12. P.P. Kumbhar and P.M. Dewang, J. Sci. Ind. Res. (India), 60, 645 (2001).

13. N. Raman, S. Thalamuthu, J.D. Raja, M.A. Neelakandan and S. Banerjee, J. Chil. Chem. Soc., 53, 1450 (2008); https://doi.org/10.4067/S0717-97072008000100025. 